\title{
An innovative electronic method for improving inpatient recruitment for bedside clinical teaching
}

\author{
Authors: Guleed Adan, Ryan Robinson, Fatima Khalid and Tom Kennedy
}

\begin{abstract}
Aims
To improve the consenting and recruitment of suitable inpatients for clinical bedside teaching; and to safely, confidentially and efficiently store and distribute this information to relevant parties.

\section{Methods}

An anonymised online questionnaire was disseminated to everyone involved in bedside teaching at the trust. The results of this questionnaire formed the basis of an innovation to identify patients with good clinical signs. Patients on admission, had an electronic 'referral' sent on the integrated clinical environment. Trainees and those involved in organising bedside teaching then accessed this list of patients on the same platform. To ensure patients were not subjected to an excessive number of examinations from trainees, the education centre at the trust issued tokens on a daily basis. This put a cap on the number of examinations a patient would take part in on any one day.
\end{abstract}

\section{Results}

We had 100 responses to our online questionnaire: $39 \%$ consultants, $36 \%$ medical students, $18 \%$ postgraduate trainees and $7 \%$ student physician associates.

The overwhelming majority of respondents (96\%) used bedside teaching for the purposes of preparing themselves or their trainees for clinical exams, eg practical assessment of clinical examination skills (PACES) or objective structured clinical examination (OSCE). A third (34\%) of respondents reported it as being difficult or very difficult to find suitable patients; they also admitted to using almost half their overall teaching time ( $49 \%$ ) to finding suitable patients. Fifty-one respondents admitted to using paper lists, and 17 respondents admitted to using WhatsApp groups or personal emails to circulate lists of patients.

\section{Conclusion}

Respondents are using half of their time to identify patients - our electronic method has been designed and implemented to recruit

Authors: Royal Liverpool and Broadgreen University Hospitals NHS Trust, Liverpool, UK patients from the point of admission to alleviate this. We found that patient information was not being efficiently or safely stored in a way that can be easily disseminated. Paper lists are inefficient as not all trainees and trainers will be able to remotely access them, while WhatsApp groups are not a secure platform for sensitive patient information. Our electronic method has resulted in a safer and more efficient alternative.

In conclusion, we have addressed the concerns of trainees and consultants in our hospital to make meaningful change through innovation on existing software platforms to safely improve the recruitment and consenting of patients for bedside teaching.

\section{Conflict of interest statement}

The authors declare no conflict of interests. 